\title{
Planejamento de enfermagem para a alta hospitalar de pacientes com insuficiência cardíaca: revisão integrativa
}

\author{
Nursing planning for hospital discharge of patients with heart failure: integrative review \\ Planificación de enfermería para el alta hospitalaria de pacientes con insuficiencia cardíaca: \\ revisión integradora
}

Recebido: 15/12/2021 | Revisado: 22/12/2021 | Aceito: 29/12/2021 | Publicado: 06/01/2022

Beatriz Costa da Silva
ORCID: https://orcid.org/0000-0001-5907-1688
Universidade Federal de Sergipe, Brasil
E-mail: enfabeatrizcosta @ gmail.com
Iara Almeida Silva
ORCID: https://orcid.org/0000-0003-3340-7037
Universidade Federal de Sergipe, Brasil
E-mail: iara17sonata @ gmail.com
Glebson Moura Silva
ORCID: https://orcid.org/0000-0002-4977-2787
Universidade Federal de Sergipe, Brasil
E-mail: glebsonmoura @yahoo.com.br
Carla Kalline Alves Cartaxo Freitas
ORCID: https://orcid.org/0000-0001-7604-9132
Universidade Federal de Sergipe, Brasil
E-mail: carlakalline@ gmail.com
Cristiane Franca Lisboa Gois
ORCID: https://orcid.org/0000-0003-4499-3756
Universidade Federal de Sergipe, Brasil
E-mail: cristianeflg@ @otmail.com
José Marcos de Jesus Santos
ORCID: https://orcid.org/0000-0001-5122-1469
Universidade de São Paulo, Brasil
E-mail: jsmarcos@ @usp.br
Onaria do Socorro Claudino Barreiro
ORCID: https://orcid.org/0000-0001-9823-4638
Universidade Federal de Sergipe, Brasil
E-mail: socorrocbarreiro@ gmail.com

\begin{abstract}
Resumo
O objetivo dessa revisão é identificar as evidências disponíveis na literatura sobre a atuação/participação da enfermagem na elaboração de um plano de alta para pacientes com IC para embasar uma dissertação de mestrado que propõe construir e validar um plano de alta para pacientes com IC, fundamentado na Teoria do Déficit do Autocuidado de Orem e Nursing Interventions Classification (NIC). A questão de pesquisa que norteou a investigação foi: Quais as evidências disponíveis na literatura sobre a atuação/participação da enfermagem na elaboração de um plano de alta para pacientes com IC? A revisão foi realizada no período de junho a agosto de 2020. Com a busca inicial nas bases de dados foi possível identificar 84 artigos primários, sendo 19 na SCOPUS, 15 no PubMed, 15 na LILACS, 12 na EmBase, 12 na BDENF, 06 na CINHAL e 05 na Web of Science. Após a leitura do título e resumo, foi possível excluir 42 estudos, restando apenas 21 para leitura na íntegra. Após essa etapa, 09 artigos restaram na amostra final. Os estudos analisados nesta revisão evidenciam a importância da enfermagem no processo de alta dos pacientes com IC, sendo possível identificar possíveis formas de atuação como: uso de cartão de alerta e fichário pessoal de saúde educativo, educação em saúde sobre a adesão a terapia medicamentosa e não medicamentosa, instigação do envolvimento do paciente e da família como ferramentas fundamentais para diminuir o número de reinternações hospitalares.
\end{abstract}

Palavras-chave: Insuficiência Cardíaca; Alta do paciente; Cuidados de enfermagem.

\begin{abstract}
The objective of this review is to identify the evidence available in the literature on the role/participation of nursing in the development of a discharge plan for patients with HF to support a master's thesis that proposes to build and validate a discharge plan for patients with HF, based on Orem's Self-Care Deficit Theory and Nursing Interventions Classification (NIC). The research question that guided the investigation was: What evidence is available in the
\end{abstract}


literature on the role/participation of nursing in the development of a discharge plan for patients with HF? The review was carried out from June to August 2020. With the initial search in the databases, it was possible to identify 84 primary articles, 19 in SCOPUS, 15 in PubMed, 15 in LILACS, 12 in EmBase, 12 in BDENF, 06 in CINHAL and 05 in Web of Science. After reading the title and abstract, it was possible to exclude 42 studies, leaving only 21 for full reading. After this step, 09 articles remained in the final sample. The studies analyzed in this review show the importance of nursing in the discharge process of patients with HF, and it is possible to identify possible ways of acting such as: use of an alert card and personal educational health file, health education on adherence to therapy drug and non-drug, instigating the involvement of the patient and family as fundamental tools to reduce the number of hospital readmissions.

Keywords: Heart Failure; Patient discharge; Nursing care.

\section{Resumen}

El objetivo de esta revisión es identificar la evidencia disponible en la literatura sobre el rol / participación de la enfermería en el desarrollo de un plan de alta para pacientes con IC para sustentar una tesis de maestría que propone construir y validar un plan de alta para pacientes con IC, según la Teoría del Déficit de Autocuidado y la Clasificación de Intervenciones de Enfermería (NIC) de Orem. La pregunta de investigación que orientó la investigación fue: ¿Qué evidencia se encuentra disponible en la literatura sobre el rol / participación de la enfermería en el desarrollo de un plan de alta para pacientes con IC? La revisión se llevó a cabo de junio a agosto de 2020. Con la búsqueda inicial en las bases de datos, fue posible identificar 84 artículos primarios, 19 en SCOPUS, 15 en PubMed, 15 en LILACS, 12 en EmBase, 12 en BDENF, 06 en CINHAL y 05 en Web of Science. Después de leer el título y el resumen, fue posible excluir 42 estudios, dejando solo 21 para la lectura completa. Después de este paso, quedaron 09 artículos en la muestra final. Los estudios analizados en esta revisión muestran la importancia de la enfermería en el proceso de alta de los pacientes con IC, y es posible identificar posibles formas de actuación como: uso de tarjeta de alerta y expediente educativo personal en salud, educación sanitaria sobre adherencia. a la terapia farmacológica y no farmacológica, instigando la implicación del paciente y su familia como herramientas fundamentales para reducir el número de reingresos hospitalarios.

Palabras clave: Insuficiencia Cardíaca; Alta del paciente; Cuidado de enfermera.

\section{Introdução}

A enfermagem tem buscado, enquanto ciência consolidar-se como uma profissão com estrutura de conhecimentos próprios que fundamentem o saber-fazer das práticas cotidianas, no processo de trabalho, a partir de elementos que caracterizem a sua identidade profissional. Por isso, tem procurado pelo sentimento de pertencimento, estabelecer a definição de competências particulares, reconhecimento, autonomia e respaldo para a tomada de decisão sobre as atribuições inerentes da profissão por meio do Processo de Enfermagem (PE) (Fernandes et al., 2018).

Nessa perspectiva, a Resolução 358/2009 dispõe sobre o PE enquanto método, que busca organizar o cuidado de enfermagem fazendo com que o processo de trabalho seja consolidado como ciência, estruturando-se em cinco etapas interrelacionadas: histórico, diagnóstico de enfermagem, planejamento, implementação e avaliação. Essa resolução ainda orienta quanto à necessidade de basear-se em uma teoria que direcione a construção de um instrumento, do planejamento das ações e intervenções de enfermagem e a escolha do diagnóstico de enfermagem, e, além disso, seja base para a avaliação dos resultados de enfermagem que forem alcançados (Cofen, 2019).

Para além da implementação do processo de enfermagem, é necessário desenvolver ferramentas que assegurem o cuidado integral. Nesse contexto, o planejamento da alta torna-se imprescindível para que a transição entre atenção hospitalar e ambulatorial seja efetiva. Vale ressaltar que o tempo em que o paciente está internado para realizar as intervenções clínicas e multidisciplinares, é de suma importância que sejam compreendidos os fatores de riscos aos quais ele está exposto, a fim de minimizar a possibilidade de readmissão hospitalar (Rohde et al., 2018).

Assim, entre as principais causas de reinternações hospitalares, destaca-se a Insuficiência Cardíaca (IC), tida como um grave problema de saúde, em virtude da falta de seguimento das orientações e o planejamento inadequado da alta hospitalar. Dessa forma, o plano de alta exige que o enfermeiro prepare o paciente e inclua os familiares, com o objetivo de prevenir agravamento do quadro clínico, controle da doença, manutenção da saúde e consequentemente promover melhor qualidade de vida desses pacientes (Weber et al., 2017). 
Deste modo, ressalta-se que a Insuficiência Cardíaca (IC) é uma síndrome complexa em que o paciente apresenta alteração da função cardíaca que culmina em sinais e sintomas de baixo débito cardíaco e/ou congestão pulmonar ou sistêmica e pode surgir aos esforços ou em repouso (Hinkle \& Cheever, 2017). É também considerada a via final de todas as patologias que acometem o coração e, por conta do seu alto índice de morbimortalidade, carece de cuidados específicos que garantam o autocuidado resolutivo (Rohde et al., 2018).

Sendo assim, a IC requer um manejo clínico de alta complexidade por parte dos profissionais, além do preparo do paciente para a alta hospitalar. Frente a isso, os enfermeiros necessitam de evidências científicas que nortearão a tomada de decisão e a sistematização da condução de enfermagem. Logo, justifica-se a relevância dessa revisão de literatura, visto que irá reunir as mais recentes evidências contidas na literatura acerca da atuação/participação dos enfermeiros no planejamento da alta hospitalar dos pacientes com IC.

Para isso teve como objetivo identificar as evidências disponíveis na literatura sobre a atuação/participação da enfermagem na elaboração de um plano de alta para pacientes com IC.

\section{Metodologia}

Trata-se de uma revisão integrativa da literatura, que de acordo com Whittemore e Knalf (2005), pode apresentar uma compreensão abrangente dos problemas relevantes para os cuidados e políticas de saúde. Foi desenvolvida de acordo com as seguintes etapas: definição da pergunta norteadora (problema); escolha dos critérios de inclusão e exclusão (seleção da amostra); seleção dos descritores, análise dos estudos primários, síntese e apresentação dos resultados.

A questão de pesquisa que norteou a investigação foi: Quais as evidências disponíveis na literatura sobre a atuação/participação da enfermagem na elaboração de um plano de alta para pacientes com IC? Para sua construção, aplicou-se a estratégia PICO (REFERE), na qual "P" (população) referiu-se aos pacientes com IC, "I" (intervenção) ao plano de alta hospitalar, "C" (comparação) não se aplicou e "O" (desfecho esperado) foi a participação da enfermagem.

Quanto aos critérios de inclusão e exclusão foram determinados considerando a questão norteadora, mediante estratégia PICO. Foram incluídos artigos contendo textos completo, nos idiomas inglês, espanhol e português, com o limite temporal final do ano 2019. Foram excluídos os estudos que tivessem outro foco que não a IC e a participação da enfermagem e que não abordasse o processo de alta hospitalar. Além disso, as teses e dissertações, além dos artigos de revisão e aqueles que não estavam disponíveis para acesso na íntegra, também foram excluídos desse estudo.

A revisão foi realizada no período de junho a agosto de 2020. As buscas foram realizadas nos meses de junho/julho por duas autoras desta revisão em sete bases de dados, sendo elas: PubMed, Web of Science, Cumulative Index to Nursing and Allied Health Literature (CINAHL), Base de Dados de Enfermagem (BDENF), Embase, Literatura Latino-Americana e do Caribe em Ciências da Saúde (LILACS) e Scopus. Definiu-se o ano 2019 como recorte temporal final da busca bibliográfica, sem delimitar o ano inicial para que fosse possível buscar o máximo de estudos.

A estratégia de busca adotou os termos dos Descritores em Ciências da Saúde (DeCS) em três idiomas e foram utilizados descritores controlados da Medical Subject Headings Section (MeSH), palavras-chave, sinônimos e operadores booleanos, conforme Tabela 1 . 
Tabela 1 - Descritores controlados e operadores booleanos cruzados nas bases de dados. São Cristóvão, SE, Brasil, 2021.

\begin{tabular}{c} 
PICO Descritores \\
P - "Insuficiência cardíaca" OR Insuficiência Cardíaca Congestiva \\
I - "Alta do paciente" OR Alta Hospitalar OR Alta do Hospital OR Planejamento da Alta \\
O - "Cuidados de Enfermagem" OR Assistência de Enfermagem OR Atendimento de enfermagem \\
\hline
\end{tabular}

Fonte: Elaborado pelos autores (2020/2021).

Quadro 1 - Estratégia de busca nas bases de dados. São Cristóvão, SE, Brasil, 2021.

\begin{tabular}{|c|c|}
\hline $\begin{array}{c}\text { Base de } \\
\text { Dados }\end{array}$ & Estratégia \\
\hline EmBase & $\begin{array}{l}\text { ('heart failure':ti,ab,kw OR 'congestive heart failure':ti,ab,kw) AND ('patient discharge':ti,ab,kw OR 'discharge, } \\
\text { patient':ti,ab,kw OR 'hospital discharge':ti,ab,kw) AND ('nursing care':ti,ab,kw OR 'management, nursing care':ti,ab,kw) }\end{array}$ \\
\hline PubMed & $\begin{array}{l}\text { ("Heart Failure" [Mesh] OR "Congestive Heart Failure") AND ("Patient Discharge" [Mesh] OR "Discharge, Patient" OR } \\
\text { "Discharge Planning") AND ("Nursing Care" }[\text { Mesh] OR "Management, Nursing Care")) }\end{array}$ \\
\hline Scopus & $\begin{array}{l}\text { ( TITLE-ABS-KEY ( "Heart Failure" OR "Congestive Heart Failure" ) AND TITLE-ABS-KEY ( "Patient Discharge" } \\
\text { OR "Discharge, Patient" OR "Discharge Planning" ) AND TITLE-ABS-KEY ( "Nursing Care" OR "Management, } \\
\text { Nursing Care" ) ) }\end{array}$ \\
\hline $\begin{array}{l}\text { Web of } \\
\text { Science }\end{array}$ & $\begin{array}{l}\text { TS=("Heart Failure" OR "Congestive Heart Failure") AND TS=("Patient Discharge" OR "Discharge, Patient" OR } \\
\text { "Discharge Planning") AND TS=("Nursing Care" OR "Management, Nursing Care") }\end{array}$ \\
\hline CINAHL & (MH "Heart Failure") AND (MH "Patient Discharge") AND (MH "Nursing Care") \\
\hline LILACS & $\begin{array}{l}\text { ("Insuficiência Cardíaca" OR "Insuficiência Cardíaca Congestiva") AND ("Alta do paciente" OR "Alta Hospitalar" OR } \\
\text { "Alta do Hospital" OR "Planejamento da Alta") AND ("Cuidados de Enfermagem" OR "Assistência de Enfermagem" OR } \\
\text { "Atendimento de Enfermagem") }\end{array}$ \\
\hline BDENF & $\begin{array}{l}\text { ("Insuficiência Cardíaca" OR "Insuficiência Cardíaca Congestiva") AND (“Alta do paciente" OR "Alta Hospitalar" OR } \\
\text { "Alta do Hospital" OR "Planejamento da Alta") AND ("Cuidados de Enfermagem" OR "Assistência de Enfermagem" OR } \\
\text { "Atendimento de Enfermagem") }\end{array}$ \\
\hline
\end{tabular}

Fonte: Elaborado pelos autores (2020/2021).

Inicialmente, foram lidos todos os títulos dos potenciais trabalhos à inclusão. Posteriormente, os estudos incluídos após leitura do título, tiveram seus resumos avaliados, sendo excluídos os duplicados e aqueles que não se enquadravam no tema. Os trabalhos selecionados até essa etapa passaram pela leitura na íntegra e foram avaliados quanto à correspondência à questão norteadora. Os artigos foram analisados pela técnica de análise temática que consistiu em leitura flutuante do conteúdo, codificação, categorização e articulação com a temática (Minayo, 2012).

Ademais, para categorizar o nível de evidência dos artigos, foi utilizado o sistema de intervenções clínicas de sete níveis: I para revisão sistemática / metanálises; II ensaio clínico randomizado; III ensaios controlados não randomizados; IV estudo de coorte ou estudos caso-controle; V metassíntese de informações qualitativas ou estudos descritivos; VI estudos qualitativos únicos ou estudos descritivos; e VII opinião de especialista (Galvão, 2006).

Assim, após a finalização das buscas nas bases, os estudos foram exportados para o Rayyan para identificação dos estudos duplicados. A leitura dos títulos e resumos deu-se através de dois revisores independentes, no qual foram selecionados 21 estudos para leitura na íntegra. Nessa etapa, houve uma concordância de $90 \%$ entre as revisoras e as eventuais discordâncias foram discutidas e avaliadas conjuntamente para se estabelecer um consenso. Sendo assim, foi possível chegar a um total de 09 estudos que responderam à pergunta norteadora.

\section{Resultados}

Com a busca inicial nas bases de dados foi possível identificar 84 artigos primários, sendo 19 na SCOPUS, 15 no PubMed, 15 na LILACS, 12 na EmBase, 12 na BDENF, 06 na CINHAL e 05 na Web of Science. Após a leitura do título e resumo, foi possível excluir 42 estudos, restando apenas 21 para leitura na íntegra. Após essa etapa, 09 artigos restaram na amostra final, sendo apresentado na Figura 1 o diagrama da seleção. 
Os dados referentes à avaliação dos estudos são apresentados a seguir (Figura 1):

Figura 1 - Fluxograma PRISMA do processo de busca de dados (Mckenzie et al., 2021). São Cristóvão, SE, Brasil. 2021.
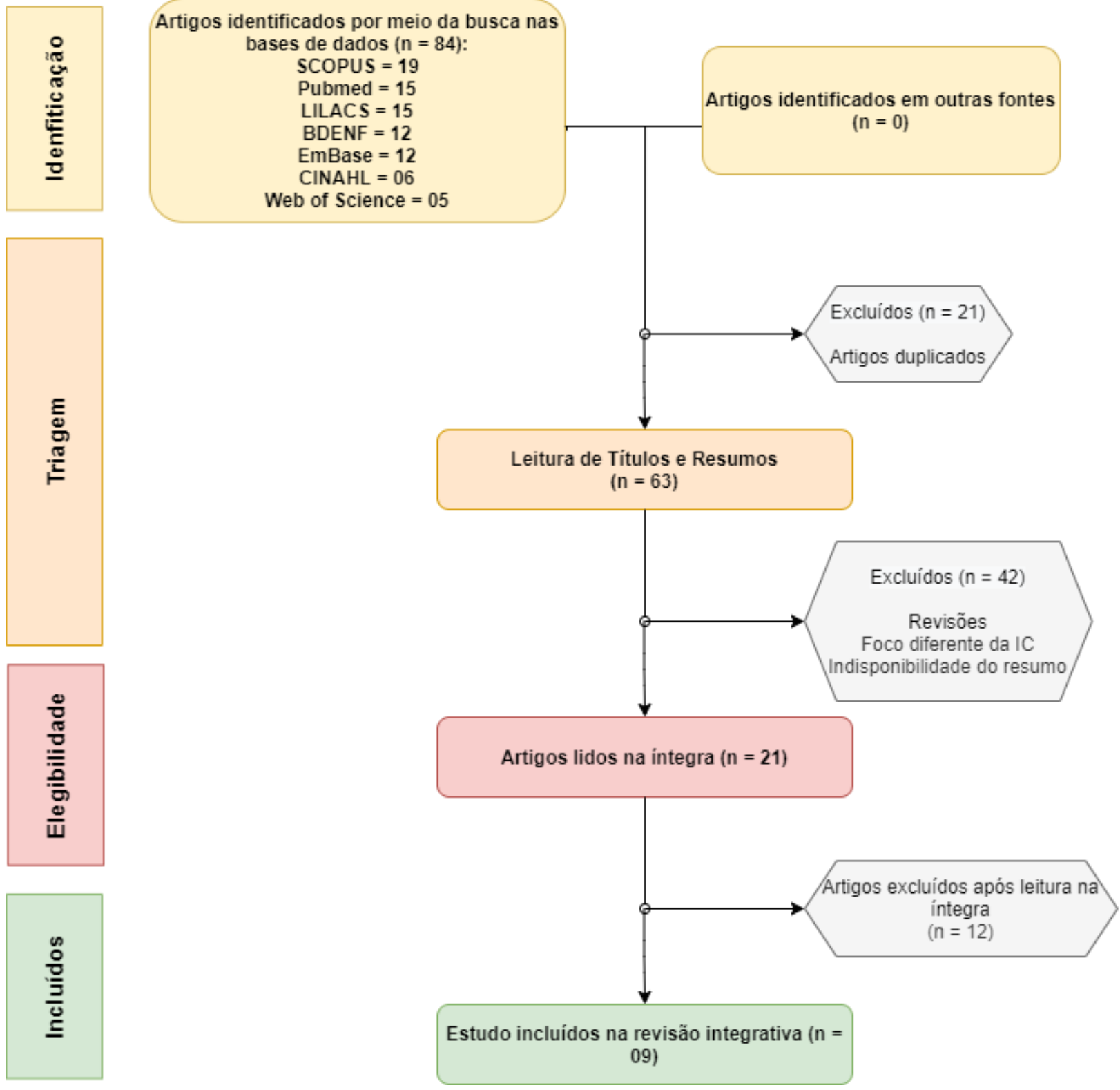

Fonte: Autores.

Os artigos foram organizados no Quadro 2, que contém as informações: Base de dados, título, local de publicação, autores/ano de publicação, delineamento do estudo, principais aspectos/resultados e nível de evidência relacionados à questão da pesquisa. 
Research, Society and Development, v. 11, n. 1, e28111124791, 2022

(CC BY 4.0) | ISSN 2525-3409 | DOI: http://dx.doi.org/10.33448/rsd-v11i1.24791

Quadro 2 - Estudos incluídos na revisão integrativa. São Cristóvão, SE, Brasil, 2021.

\begin{tabular}{|c|c|c|c|c|c|c|}
\hline $\begin{array}{l}\text { Base de } \\
\text { dados }\end{array}$ & Título & Autores/ano & País & $\begin{array}{c}\text { Delineamento do } \\
\text { estudo }\end{array}$ & Principais aspectos/resultados relevantes evidenciados & $\begin{array}{c}\text { Nível de } \\
\text { evidênci } \\
\mathbf{a}\end{array}$ \\
\hline EmBase & $\begin{array}{l}\text { Hospital readmission rates } \\
\text { among disadvantaged heart } \\
\text { failure patients enrolled in } \\
\text { outreach program }\end{array}$ & $\begin{array}{l}\text { Kira } \\
\text { McClinton et } \\
\text { al., } 2015 .\end{array}$ & EUA & Estudo transversal & $\begin{array}{l}\text { Os pacientes inscritos no programa ACTIVATE } \\
\text { receberam cuidados transitórios na alta e uma visita } \\
\text { domiciliar dentro de } 48 \text { horas após a alta. No entanto, foi } \\
\text { identificada uma taxa de readmissão substancialmente } \\
\text { maior nos pacientes com IC com comorbidades crônicas } \\
\text { do que para pacientes sem IC. }\end{array}$ & V \\
\hline EmBase & $\begin{array}{l}\text { The quality of hospital } \\
\text { work environments and } \\
\text { missed nursing care is } \\
\text { linked to heart failure } \\
\text { readmissions: A cross- } \\
\text { sectional study of US } \\
\text { hospitals }\end{array}$ & $\begin{array}{l}\text { J Margo } \\
\text { Brooks } \\
\text { Carthon et al., } \\
2015 .\end{array}$ & EUA & Estudo transversal & $\begin{array}{l}\text { Os resultados revelam uma associação entre os cuidados } \\
\text { de enfermagem que não são prestados e reinternações por } \\
\text { insuficiência cardíaca. Cerca de } 40 \% \text { dos enfermeiros } \\
\text { relataram não conseguir conversar com seus pacientes } \\
\text { durante o último contato anterior à alta. }\end{array}$ & V \\
\hline Pubmed & $\begin{array}{l}\text { Hospital nurses' comfort in } \\
\text { and frequency of } \\
\text { delivering heart failure } \\
\text { self-care education }\end{array}$ & $\begin{array}{l}\text { Nancy M } \\
\text { Albert et al., } \\
2014 .\end{array}$ & EUA & Estudo descritivo & $\begin{array}{l}\text { O conforto dos enfermeiros em fornecer educação sobre } \\
\text { autocuidado com insuficiência cardíaca foi associado à } \\
\text { frequência de fornecimento de educação a pacientes } \\
\text { hospitalizados. Quando as enfermeiras sentiam-se } \\
\text { confortáveis orientando pacientes com IC na área do } \\
\text { autocuidado ou para um item específico, ainda era abaixo } \\
\text { dos níveis de conforto da enfermeira. Os pacientes com } \\
\text { insuficiência cardíaca têm no autocuidado uma das áreas } \\
\text { com maior probabilidade de estabilizar ou melhorar o } \\
\text { estado clínico. }\end{array}$ & V \\
\hline Pubmed & $\begin{array}{l}\text { The role of patient-held } \\
\text { alert cards in promoting } \\
\text { continuity of care for Heart } \\
\text { Failure Patients }\end{array}$ & $\begin{array}{l}\text { Anne } \\
\text { McBrideet } \\
\text { al., } 2014 .\end{array}$ & $\begin{array}{l}\text { Reino } \\
\text { Unido }\end{array}$ & Estudo descritivo & $\begin{array}{l}\text { Este projeto confirma que os cartões de alerta acerca do } \\
\text { quadro de IC retidos pelo paciente podem contribuir para } \\
\text { a continuidade do cuidado, além de sinalizar acerca das } \\
\text { necessidades imediatas de emergência tradicionalmente } \\
\text { associadas a eles. Podem melhorar a comunicação e a } \\
\text { coordenação entre a equipe do hospital e capacitar os } \\
\text { pacientes e seus familiares, para o pós-alta. }\end{array}$ & V \\
\hline Pubmed & $\begin{array}{l}\text { Emergency Nurses' } \\
\text { Perceived Confidence in } \\
\text { Participating in the } \\
\text { Discharge Process of } \\
\text { Congestive Heart Failure } \\
\text { Patients From the } \\
\text { Emergency Department: A } \\
\text { Quantitative Study } \\
\end{array}$ & $\begin{array}{l}\text { Alsacia L } \\
\text { Sepulveda- } \\
\text { Pacsi, } 2019 .\end{array}$ & EUA & Estudo transversal & $\begin{array}{l}\text { A principal descoberta é que apenas uma minoria dos } \\
\text { enfermeiros se sentiu "muito" confiante em sua } \\
\text { capacidade de desempenhar várias funções com esses } \\
\text { pacientes antes da alta (com exceção do monitoramento } \\
\text { de peso). Mais de dois terços dos enfermeiros } \\
\text { responderam que orientações específicas sobre a alta os } \\
\text { ajudaria a se sentirem mais confiantes, como por exemplo, } \\
\text { se houvesse diretrizes específicas sobre o processo de alta. }\end{array}$ & V \\
\hline Scopus & $\begin{array}{l}\text { Reducing Readmissions } \\
\text { among Heart Failure } \\
\text { Patients Discharged to } \\
\text { Home Health Care: } \\
\text { Effectiveness of Early and } \\
\text { Intensive Nursing Services } \\
\text { and Early Physician } \\
\text { Follow-Up } \\
\end{array}$ & $\begin{array}{l}\text { Christopher } \\
\text { M. Murtaugh, } \\
\text { Partha Deb et } \\
\text { al., } 2017 .\end{array}$ & EUA & $\begin{array}{l}\text { Estudo } \\
\text { observacional } \\
\text { retrospectivo }\end{array}$ & $\begin{array}{l}\text { Os resultados indicam que a combinação de serviços de } \\
\text { enfermagem domiciliar precoce e intensiva e pelo menos } \\
\text { uma consulta de acompanhamento médico na semana } \\
\text { após a alta hospitalar tem o potencial de reduzir o risco de } \\
\text { readmissão para uma parte substancial dos pacientes com } \\
\text { IC do grupo do estudo que receberam alta aos cuidados de } \\
\text { saúde ao domicílio. }\end{array}$ & IV \\
\hline Scopus & $\begin{array}{l}\text { Person-centred care - An } \\
\text { approach that improves the } \\
\text { discharge process }\end{array}$ & $\begin{array}{l}\text { Kerstin Ulin } \\
\text { et al., } 2016 .\end{array}$ & Suécia & $\begin{array}{l}\text { Estudo clínico } \\
\text { randomizado }\end{array}$ & $\begin{array}{l}\text { O principal achado no presente estudo foi a diferença no } \\
\text { tempo dos componentes importantes do processo de alta } \\
\text { entre o protocolo empregado e os cuidados habituais. Este } \\
\text { melhora os processos de alta porque os pacientes são } \\
\text { vistos como pessoas competentes para se envolver no } \\
\text { planejamento de seus cuidados. O presente achado } \\
\text { contribui para as evidências crescentes de que o modelo } \\
\text { de atenção centrada na pessoa é mais eficaz do que o } \\
\text { tratamento usual. }\end{array}$ & III \\
\hline $\begin{array}{l}\text { Web of } \\
\text { Science }\end{array}$ & $\begin{array}{l}\text { Patient readiness for } \\
\text { hospital discharge and its } \\
\text { relationship to discharge } \\
\text { preparation and structural } \\
\text { factors: A cross-sectional } \\
\text { study }\end{array}$ & $\begin{array}{l}\text { Cedric } \\
\text { Mabire et al., } \\
2019 .\end{array}$ & Suíça & Estudo transversal & $\begin{array}{l}\text { A prontidão do paciente para as avaliações de alta } \\
\text { hospitalar foi maior em pacientes que receberam } \\
\text { intervenções de ensino de alta, em unidades cujas } \\
\text { enfermeiras tinham altos níveis gerais de experiência } \\
\text { profissional e onde os pacientes forneceram auto } \\
\text { avaliações melhores de seu estado de saúde. }\end{array}$ & V \\
\hline Lilacs & $\begin{array}{l}\text { Patient readiness for } \\
\text { hospital discharge and its } \\
\text { relationship to discharge } \\
\text { preparation and structural } \\
\text { factors: A cross-sectional } \\
\text { study }\end{array}$ & $\begin{array}{l}\text { Souza, } \\
\text { Patrícia Maria } \\
\text { Barreto Bellot } \\
\text { de; Queluci, } \\
\text { Gisella de } \\
\text { Carvalho; } \\
\text { 2014. }\end{array}$ & Brasil & Estudo descritivo & $\begin{array}{l}\text { É enfatizada a necessidade do planejamento da alta } \\
\text { hospitalar de forma sistematizada, integral, organizada e } \\
\text { flexível, destacando as ações da equipe multidisciplinar, } \\
\text { com a coordenação dos cuidados pelos enfermeiros e que } \\
\text { este planejamento inicie no momento da internação dos } \\
\text { pacientes, perdurando por toda hospitalização destes e, se } \\
\text { necessário, continuado também para o contexto familiar. }\end{array}$ & V \\
\hline
\end{tabular}

Fonte: Elaborado pelos autores (2020/2021). 
Dentre os artigos encontrados, os anos de publicação variavam entre 2014 e 2019, sendo 33,3\% do ano 2014, 22,2\% para os anos 2015 e 2019, 11,1\% nos anos 2016 e 2017 e em 2018 não foram encontrados artigos publicados acerca da temática. Com relação à distribuição entre os países de realização dos estudos, a maioria deles foi realizada nos EUA (55,6\%), seguidos do Reino Unido, Suécia, Suíça e Brasil (11,1\% cada).

\section{Discussão}

As evidências encontradas neste estudo consistem na síntese referencial para enfermeiros que buscam estratégias no desenvolvimento de um plano de alta direcionadas aos pacientes com IC, dentre elas há a utilização de fichário pessoal de saúde com material educativo, melhoria dos cuidados de enfermagem para impacto na redução de reinternações, utilização de cartão de alerta mantido pelo paciente para facilitar a comunicação entre a rede hospitalar e outros serviços, além de dar embasamento aos profissionais através de diretrizes específicas sobre o processo de alta. Cada um dos nove estudos selecionados passa a ser objeto dessa discussão.

De acordo com a Sociedade Brasileira de Cardiologia (SBC), a admissão hospitalar por descompensação de IC constitui um momento crítico na história natural da doença, representando um desafio para equipe de saúde a nível hospitalar e ambulatorial. Nesse contexto, o planejamento de alta e a transição de cuidados precisam ter início antes mesmo da alta propriamente dita, devendo a equipe planejar e estruturar esse momento assim que o paciente é admitido (Rohde et al., 2018).

Sendo assim, foi identificado um único estudo que abordou os três elementos essenciais para elaboração do plano de alta que envolve a avaliação e planejamento das necessidades de alta; ensino de alta (ações educacionais); e gerenciamento de alta (apoio pós-alta necessário). Para os autores, há fatores que refletem em maior agilidade para a alta hospitalar, são eles: enfermeiros mais experientes, o preparo do autocuidado do paciente e a coordenação dos principais sintomas da IC refletem em maior agilidade para alta hospitalar (Mabire et al., 2019).

Tais evidências corroboram com o modelo proposto pela Diretriz Brasileira de Insuficiência Cardíaca Crônica e Aguda para sistematização do cuidado na alta hospitalar, sendo o processo classificado em três etapas, conforme cores: vermelha, amarela e verde. Temos que a cor vermelha se refere aos pré-requisitos clínicos, em que se busca atingir o objetivo de compensação da doença, além de atingir o melhor equilíbrio hemodinâmico possível; na amarela encontram-se os prérequisitos multidisciplinares com a finalidade de evitar outras hospitalizações, são eles a educação do paciente, orientações sobre estilo de vida, autocuidado e adesão ao tratamento; e na verde está o plano de seguimento ambulatorial, com retorno agendado (Rohde et al., 2018).

No tocante ao plano de alta, encontram-se na literatura estudos que evidenciam quanto à necessidade deste ser individualizado e também incluir a família do paciente. Ressalta-se ainda que é um processo que deve ser iniciado já na admissão do usuário no serviço de saúde e desenvolvido durante todo o período da internação. E complementam que a existência de um plano de alta padronizado na instituição aumenta a eficácia e qualidade do cuidado prestado (Suzuki, Carmona \& Lima, 2011), (Miasso \& Cassiani, 2003), (Teixeira, Rodrigues \& Machado, 2012), (Paiva \& Valadares, 2013), (Reis et al., 2013).

Esse cuidado sistematizado demanda que os profissionais enfermeiros detenham conhecimento de um arcabouço teórico específico que embase as habilidades intelectuais de técnicas e criticidade, que objetive identificar e documentar os cuidados de forma padronizada através de terminologias padrões e que vincule os elementos essenciais para a prática profissional (Menezes et al., 2020).

Os sistemas de classificação e linguagem padronizada podem ser utilizados como ferramentam que auxiliam e qualificam o processo de enfermagem o que reflete diretamente no cuidado ao usuário. Nesse contexto, os sistemas NANDA e 
NIC são utilizados em diferentes instituições de saúde no mundo inteiro e demonstram eficiência e eficácia além de fácil adaptação por parte dos profissionais (Boeira, Sartori \& Baltazar, 2020).

Além disso, foi possível observar no presente estudo que as principais recomendações ou estratégias realizadas pelos enfermeiros concentram-se na adesão a terapia medicamentosa, restrição hídrica de 1,0 a 1,5 litros/dia, dieta hipossódica (2 a 3 gramas/dia); controle do peso diário; o não uso de medicamentos sem prévia orientação da equipe e prescrição médica; reconhecimento dos sinais e sintomas de descompensação e piora da doença; vacinação anti-influenza e pneumocócica, monitoramento da pressão arterial (Souza \& Queluci, 2014), (Mcclinton et al, 2015). No entanto, foi percebida fragilidade no que concerne a prescrição de cuidados de enfermagem nos estudos encontrados. O que reforça a importância de utilizar efetivamente o processo de enfermagem na prática.

Outra observação importante e considerada básica no preparo da alta hospitalar identificada nesse estudo refere-se ao fornecimento de informações claras e objetivas aos pacientes e seus familiares, sendo ressaltada a importância de envolver o paciente no seu autocuidado (Souza \& Queluci, 2014). Cabe destacar que as ações educacionais e os recursos utilizados no planejamento da alta são cruciais no processo de cuidado e podem gerar impactos positivos ou negativos, a depender de como são trabalhadas diante do nível de compreensão e necessidades de cada paciente.

Há autores que trazem que a prática do autocuidado na IC está intrinsecamente relacionada aos comportamentos das pessoas para manter a sua saúde e também às decisões que tomam quando ocorre a piora dos sintomas. Além disso, a manutenção do autocuidado engloba a adesão às recomendações farmacológicas, cessação do uso de tabaco, consumo de dieta com pouco sal, monitoração de peso e de sinais ou sintomas de descompensação diariamente e consumo limitado de álcool. Sendo assim, autocuidado é uma tomada de decisão na escolha de comportamentos que mantém a estabilidade do quadro clínico e, também, a resposta aos sintomas quando ocorrem (Da Conceição et al., 2015).

Nessa perspectiva, observa-se a importância do cuidado integral e de estar embasado na segurança e bem-estar dos pacientes com IC. Assim, é fundamental que todas as orientações sejam realizadas com o intuito de preparar o paciente para a alta de forma contínua, adequada e efetiva. Além disso, reforça quanto à relevância de englobar os familiares nesse processo de forma clara e objetiva, o que permitirá a continuação e sucesso do tratamento no ambiente domiciliar (Souza \& Queluci, 2014).

Ao que concerne à abordagem do cuidado em equipe, três estudos mencionaram a importância do planejamento da alta hospitalar sistematizada e organizada, salientando as ações da equipe multidisciplinar com ênfase no trabalho da enfermagem como mecanismo fundamental para o cuidado dos pacientes acometidos pela insuficiência cardíaca (Souza \& Queluci, 2014), (Ulin et al, 2016), (Sepulveda-Pacsi, 2019).

O planejamento da alta consiste na criação de um plano para os pacientes que considere as suas individualidades, objetivando diminuir os custos com reinternações, melhorar resultados clínicos e garantir o seguimento do tratamento e que os cuidados após a alta sejam organizados (Gonçalves-Bradley et al., 2016).

Sendo assim, é importante que o plano de alta seja organizado de forma multidimensional e pondere aspectos físicos, psicológicos, doença de base, limitações físicas e motoras, rede de apoio, terapia medicamentosa e autonomia individual, de modo que, possibilite uma alta hospitalar bem sucedida (Galvin et al., 2017). Os resultados desse estudo apontam para a redução dos índices de reinternação em locais em que há diretrizes específicas sobre o processo de alta e em que há melhoria dos cuidados de enfermagem, além da utilização de instrumentos com materiais educativos para os pacientes e familiares.

Um dos estudos analisados evidenciou que as altas taxas de readmissões ocorreram em virtude de educação inadequada do paciente e falta de adesão aos medicamentos. $\mathrm{O}$ autor destaca que grande parte das enfermeiras que participaram da pesquisa destacou a necessidade de diretrizes específicas sobre o processo de alta com pacientes com insuficiência cardíaca. Revelou-se ainda, que cuidados direcionados por enfermeiras melhoram as disparidades nas taxas de readmissão (Sepulveda-Pacsi, 2019). 
Enfatiza-se ainda quanto à importância de adaptar diretrizes desenvolvidas para delinear os critérios para a alta do paciente com IC que tenha sido estabilizado no pronto-socorro, visto que isso aumentará a confiança dos enfermeiros que conduzirão esse processo de alta (Sepulveda-Pacsi, 2019). Sendo que melhorar essa autoconfiança impacta diretamente na assistência prestada, visto que há estudos que descobriram que o cuidado liderado por profissionais enfermeiros melhoram as disparidades nas taxas de readmissão para diversas condições, inclusive a insuficiência cardíaca (Davis et al., 2007); (O'connell et al., 2001).

Outro estudo mostrou que $40 \%$ dos enfermeiros referem não conseguir orientar seus pacientes durante o último turno em decorrência das condições de trabalho desfavoráveis. Além disso, também enfatiza que uma série de fatores influenciam as readmissões por insuficiência cardíaca, incluindo descompensação da função renal, altas taxas de comorbidades, baixa adesão ao tratamento instituído, os estressores psicossociais aos quais os pacientes estão expostos e limitações funcionais (Carthon et al., 2015).

Por isso, destaca-se a importância de o profissional investir na educação continuada com enfoque nas ações multidisciplinares envolvendo o paciente e sua família. Já se sabe que um dos motivos de reinternações é justamente a falta de preparo adequado do usuário e familiares para o momento da alta hospitalar. Para isso, deve ser incentivado o permanente treinamento das equipes acerca dos aspectos fundamentais de saúde para o cuidado do paciente com IC (Souza \& Queluci, 2014).

Também se destaca nessa revisão a articulação entre assistência terciária e a atenção primária, pois os pacientes com IC pós-aguda podem deteriorar rapidamente se não seguirem os cuidados clínicos prescritos. Esse estudo afirma que a combinação de serviços de enfermagem precoce e intensiva e pelo menos uma consulta de acompanhamento médico na semana após a alta hospitalar, têm o potencial de reduzir de forma significativa as reinternações hospitalares dos pacientes com IC (Murtaugh et al., 2017). Deste modo a continuidade do cuidado torna-se imprescindível na assistência desses pacientes.

Sendo assim, é através do planejamento da alta hospitalar que se reforça a necessidade de estudar e implementar estratégias que influenciem na consolidação das atividades de continuidade do cuidado e melhoria na transição da assistência para outro ponto da Rede de Atenção à Saúde (RAS). Pode-se destacar a atuação dos enfermeiros como facilitadores desse processo, visto que possuem, em geral, maior vínculo com os usuários nos diferentes cenários de saúde e agregam conhecimentos e práticas. Sendo assim, o plano de alta planejado e sistematizado com a participação da equipe de enfermagem, do paciente e dos seus familiares/cuidadores visa alcançar melhores resultados em saúde por compartilhar saberes para esse fim (Ribas et al., 2018).

No presente estudo, observou-se a importância do direcionamento da atuação do enfermeiro integrado com a equipe multidisciplinar para efetivar um processo da alta hospitalar amparado por referenciais teóricos próprios, a fim de refletir diretamente na continuidade efetiva do tratamento no ambiente domiciliar, o que impacta na estabilização do quadro clínico e por consequência melhoria do bem-estar do paciente com insuficiência cardíaca.

\section{Conclusão}

Os estudos analisados nesta revisão evidenciam a importância da enfermagem no processo de alta dos pacientes com IC, sendo possível identificar possíveis formas de atuação como: uso de cartão de alerta e fichário pessoal de saúde educativo, educação em saúde sobre a adesão a terapia medicamentosa e não medicamentosa, instigar o envolvimento do paciente e da família como ferramentas fundamentais para diminuir o número de reinternações hospitalares. Cabe ainda destacar, a relevância da equipe de enfermagem estar inserida na equipe multiprofissional, bem como a comunicação intersetorial para implementação de um plano de alta resolutivo.

Dentre as limitações do estudo, há a ausência de estudos amplamente divulgados que evidenciem a utilização dos 
sistemas de classificação e linguagem padronizada na enfermagem, o que da margem a elaboração de novos estudos sobre esse assunto relevante para a prática de enfermagem. Ressalta-se ainda, a necessidade do desenvolvimento de novos estudos com níveis de evidência maiores que os encontrados no presente estudo, com o intuito de fornecer suporte científico à assistência de enfermagem. Podem ser elaborados futuros estudos acerca de possíveis plano de alta de enfermagem, bem como identificar a viabilidade da aplicabilidade destes nos serviços de saúde.

\section{Referências}

Boeira, S., Sartori, R., \& Baltazar, E. M. (2020). "Educação permanente para qualificação do processo de enfermagem com o uso de terminologia padronizada de enfermagem. Editora Científica Digital.

Carthon, J. M. B., Lasater, K. B., Sloane, D. M., \& Kutney-Lee, A. (2015). A qualidade dos ambientes de trabalho hospitalares e a falta de cuidados de enfermagem estão relacionadas às reinternações por insuficiência cardíaca: um estudo transversal de hospitais dos Estados Unidos. BMJ Quality \& Safety, 24 (4), 255-263.

Conselho Federal De Enfermagem (COFEN). (2009). Resolução COFEN-358/2009. http://www.cofen.gov.br/resoluo-cofen-3582009_4384.html

Davis, A. M., et al. (2007) Disparidades de saúde cardiovascular: uma revisão sistemática das intervenções de saúde. Pesquisa e revisão de assistência médica, 64 (5 Supl.), 29S - 100S.

de Souza, P. M. B. B., \& de Carvalho Queluci, G. (2014). The art of caring for patients with heart failure at hospital discharge: considerations for nursing healthcare practice. Revista de Pesquisa Cuidado é Fundamental Online, 6(1), 153-167.

Dochterman, J. M.; Bulechek, G. M., \& Butcher, H. K. (2016) Classificação das intervenções de enfermagem (NIC). (6a ed.), Elsevier.

Fernandes, M. C., Silva, L. M. S. D., Silva, M. R. F. D., Torres, R. A. M., Dias, M. S. D. A., \& Moreira, T. M. M. (2018). Identity of primary health care nurses: perception of" doing everything". Revista brasileira de enfermagem, 71, 142-147.

Galvão, C. M. (2006) Níveis de evidência. Acta Paul Enferm. 19(2):V.

Galvin, E. C., Wills, T., \& Coffey, A. (2017). Readiness for hospital discharge: a concept analysis. Journal of advanced nursing, 73(11), $2547-2557$.

Gonçalves-Bradley, D. C., Lannin, N. A., Clemson, L. M., Cameron, I. D., \& Shepperd, S. (2016). Discharge planning from hospital. Cochrane database of systematic reviews, (1).

Hinkle, J. L; \& Cheever, K. H. (2017) Brunner e Suddarth: Tratado de Enfermagem médico-cirúrgica. (13a ed.), Guanabara-Koogan.

Mabire, C., Bachnick, S., Ausserhofer, D., Simon, M., \& Match RN Study Group. (2019). Patient readiness for hospital discharge and its relationship to discharge preparation and structural factors: A cross-sectional study. International journal of nursing studies, 90, 13-20.

McClinton, K., Garganta, M., Lato, M., Martin, L., Kilby, S., \& Kalya, A. (2015). Hospital Readmission Rates Among Disadvantaged Heart Failure Patients Enrolled in Outreach Program. Journal of Cardiac Failure, 21(8), S79.

Menezes, H. F. D., Camacho, A. C. L. F., Lins, S. M. D. S. B., Campos, T. D. S., Lima, F. R., Jales, A. K. F. A., \& Silva, R. A. R. D. (2020). Termos da linguagem especializada de enfermagem para pacientes renais crônicos em tratamento conservador. Revista Brasileira de Enfermagem, 73.

Miasso, A. I., \& Cassiani, S. H. D. B. (2005). Administração de medicamentos: orientação final de enfermagem para a alta hospitalar. Revista da Escola de Enfermagem da USP, 39, 136-144.

Minayo, M. C. D. S. (2012). Análise qualitativa: teoria, passos e fidedignidade. Ciência \& saúde coletiva, 17, 621-626.

Mckenzie J. E., et al. (2021). A declaração PRISMA 2020: uma diretriz atualizada para relatar revisões sistemáticas. International Journal of Surgery. 88: 105906.

O'Connell, A. M., Crawford, M. H., \& Abrams, J. (2001). Heart failure disease management in an indigent population. American Heart Journal, 141(2), 254258.

Paiva, R. S. D., \& Valadares, G. V. (2013). Vivenciando o conjunto de circunstâncias que influenciam na significação da alta hospitalar: estudo de enfermagem. Escola Anna Nery, 17, 249-255.

Ribas, E. D. N., Bernardino, E., Larocca, L. M., Poli, P., Aued, G. K., \& Silva, C. P. C. D. (2018). Nurse liaison: a strategy for counter-referral. Revista brasileira de enfermagem, 71, 546-553.

Rohde, L. E. P., Montera, M. W., Bocchi, E. A., Clausell, N. O., Albuquerque, D. C. D., Rassi, S., \& Martins, W. D. A. (2018). Diretriz brasileira de insuficiência cardíaca crônica e aguda. Arquivos Brasileiros de Cardiologia, 111(3), 436-539.

Sepulveda-Pacsi, AL (2019). A percepção de confiança dos enfermeiros de emergência em participar do processo de alta de pacientes com insuficiência cardíaca congestiva do pronto-socorro: Um estudo quantitativo. Hispanic Health Care International, 17 (1), $30-35$.

Suzuki, V. F., Carmona, E. V., \& Lima, M. H. M. (2011). Planejamento da alta hospitalar do paciente diabético: construção de uma proposta. Revista da Escola de Enfermagem da USP, 45, 527-532. 
Research, Society and Development, v. 11, n. 1, e28111124791, 2022

(CC BY 4.0) | ISSN 2525-3409 | DOI: http://dx.doi.org/10.33448/rsd-v11i1.24791

Teixeira, J. P. D. D. S., Rodrigues, M. C. S., \& Machado, V. B. (2012). Educação do paciente sobre regime terapêutico medicamentoso no processo de alta hospitalar: uma revisão integrativa. Revista Gaúcha de Enfermagem, 33, 186-196.

Ulin, K., Olsson, L. E., Wolf, A., \& Ekman, I. (2016). Person-centred care-An approach that improves the discharge process. European Journal of Cardiovascular Nursing, 15(3), e19-e26.

Weber, L. A. F., Lima, M. A. D. D. S., Acosta, A. M., \& Marques, G. Q. (2017). Transição do cuidado do hospital para o domicílio: revisão integrativa. Cogitare enfermagem. 22(3), e47615.

Whittemore, R., \& Knafl, K. (2005). The integrative review: updated methodology. Journal of advanced nursing, 52(5), 546-553. 